\title{
Performance Evaluation of Channel Switching Scheme for Packet Data Transmission in Radio Network Controller
}

\author{
Yoshiaki Ohta, Kenji Kawahara, Takeshi Ikenaga, and Yuji Oie \\ Dept. of Computer Science and Electronics, Kyushu Institute of Technology \\ Iizuka, Fukuoka 820-8502, Japan \\ yoshiaki@infonet.cse.kyutech.ac.jp, \{kawahara, ike, oie\}@cse.kyutech.ac.jp
}

\begin{abstract}
W-CDMA (Wideband-CDMA) is expected for the radio access technology of the third-generation mobile telecommunication systems. In the second-generation systems, voice traffic from each user has mainly been transmitted via the dedicated transport (radio) channel. In addition, the thirdgeneration systems will efficiently accommodate data traffic based on the packet transmission in the shared common transport channel. Therefore, data traffic can be transmitted via one of two types of channels: i.e., dedicated channel and common channel. However, the channel selecting/switching scheme in RNC (Radio Network Controller) has not been standardized. Thus, in the present paper, we will propose some channel switching schemes and evaluate their performance in terms of the packet loss probability and the utilization of dedicated channels by means of simulations.
\end{abstract}

Keywords: IMT-2000, W-CDMA, RNC, Dedicated Channel, Common Channel

\section{Introduction}

Wireless communications have been recently attracted and spread widely with the rapid growth of the Internet. In the second-generation mobile telecommunication systems, the major services are limited to basic services such as voice, facsimile, and low-rate-data transmission. In the third-generation mobile telecommunication systems, a variety of services such as high speed Internet access, multimedia data transmission, and global roaming will be expected. For that reason, the ITU (International Telecommunication Union) began its studies on a global standard for mobile telecommunication systems, which is referred to as IMT-2000 (International Mobile Telecommunications-2000) [1][2][3]. In the third-generation mobile telecommunication systems, the future radio transmission technology is strongly expected to efficiently transmit not only legacy voice traffic but also the data traffic based on the packet transmission. Thus, a lot of proposals for radio transmission technology candidates have been submitted to the ITU. Most of them were based on CDMA (Code Division Multiple Access) but with differences in technical details, and to prevent multiple standard problems, they have been integrated and developed to some global standards. Especially in them, W-CDMA (Wideband-CDMA) [4][5] receives much attention for the radio transmission technology and most research has been done in this area. The standard of W-CDMA is defined in detail by 3GPP (3rd Generation Partnership Project) [6], and throughout the standard process, it discussed the packet transmission (radio) channel structure and proposed one method. In 


\begin{tabular}{|c|c|c|c|c|c|c|c|c|c|}
\hline & $\begin{array}{c}\text { Wireless } \\
\text { Links }\end{array}$ & & \multicolumn{2}{|c|}{ UTRAN } & & & \multicolumn{2}{|r|}{$\mathrm{CN}$} & $\begin{array}{l}\text { External } \\
\text { Networks } \\
\end{array}$ \\
\hline TCP/UDP & & & & & & & & & TCP/UDP \\
\hline IP & & & & & & & IP & IP & IP \\
\hline PDCP & & & $\mathrm{PDCl}$ & \begin{tabular}{|l|} 
GTP \\
\end{tabular} & GTP & GTP & GTP & & \\
\hline RLC & & & 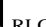 & UDP & UDP & UDP & UDP & 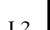 & $\mathrm{L} 2$ \\
\hline MAC & & & MAC & L2 & L2 & L2 & L2 & & \\
\hline L1 & CDMA & & L1 & L1 & L1 & L1 & $\mathrm{L} 1$ & $\mathrm{~L} 1$ & L1 \\
\hline UE & & Node B & & & SG & & & $\mathrm{SN}$ & Server \\
\hline
\end{tabular}

Fig. 1. Protocol stacks of W-CDMA packet data services

the method, two channels are mainly provided for the packet transmission: dedicated channel and common channel. For example, in the case that a large amount of traffic is transmitted by some flow, a channel will be dedicated to it and its packets can be efficiently transmitted over it, whereas flows with only a small amount of traffic share the common channel. Radio resources can be efficiently utilized in some adaptive way of selecting an appropriate transmission channel according to the traffic characteristics [7]. The channel selecting/switching is controlled by RNC (Radio Network Controller), and the specific scheme in RNC has not been standardized.

Therefore, our major interest in the present paper is to clarify the issues related to channel selecting/switching schemes and study their characteristics. We will thus propose specific channel selecting/switching schemes in accordance with current W-CDMA specifications and evaluate their performance in terms of the packet loss probability and the utilization of dedicated channels by means of simulations. In addition, we will discuss their characteristics based upon performance comparison.

\section{Architecture of RNC}

Fig.1]shows an example of protocol stacks in W-CDMA packet data services [6]. Several nodes exist between Internet servers and UE (User Equipment) or mobile terminals. In CN (Core Network), two distinct elements exist: GGSN (Gateway General packet radio service Support Node) and SGSN (Serving General packet radio service Support Node). GGSN is the switch at the point where nodes are connected to external networks. All incoming and outgoing packets must go through GGSN. SGSN is the database that serves UE in its current location, and provides the function of packet switching and routing. In UTRAN (Universal Terrestrial Radio Access Network) which handles radio-related functions, two distinct elements also exist: RNC and Node B. RNC is responsible for managing radio resources in wireless links. Node B corresponds to a base station (BS) which handles the radio communication over W-CDMA air interfaces. In these protocol stacks, RNC includes the physical layer and the link layer, and it segments packets received from external networks into several data blocks of fixed length, recovers transmission errors that occurred in wireless links, and assigns proper transmission channels. These functions are provided in RLC (Radio Link Control)/MAC (Media Access Control) layers described in RNC. 


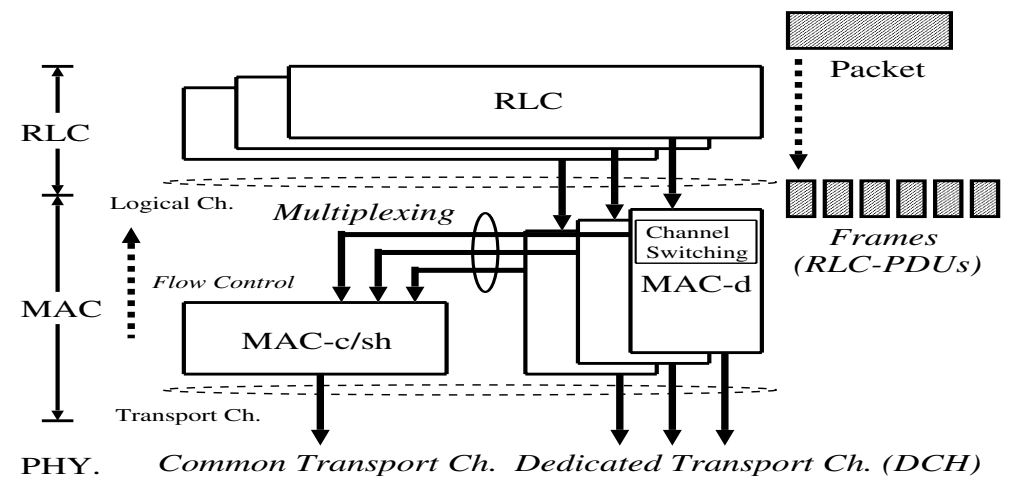

Fig. 2. The architecture of RLC/MAC layers

\subsection{Architecture of RLC/MAC Layers}

Fig. 2 illustrates the architecture of RLC/MAC layers as well as a data transmission method used in it. In the RLC layer, packets transmitted from external networks are segmented into RLC PDUs, i.e., link layer frames, they are then forwarded to the RLC dedicated buffer for each flow. Thus, the transmission unit in RLC/MAC layers is the frame, to which control information such as its sequence number is added in the header. To achieve good performance even in high error rate links, an ARQ (Automatic Repeat reQuest) mechanism based on the frame is provided for protecting against transmission errors through a limited number of retransmission attempts. An ARQ protocol is implemented in W-CDMA packet data services. This protocol is based on the selective repeat scheme.

In the MAC layer, mainly two sublayers are described: MAC-d sublayer and MAC$\mathrm{c} / \mathrm{sh}$ sublayer. The main function of the MAC-d sublayer is channel selecting/switching and control of dedicated channels. The main function of the MAC-c/sh sublayer is control of common channels. One MAC-d sublayer in RNC is allocated for each UE, while only one MAC-c/sh in RNC is shared by all UE in a cell.

In RLC/MAC layers, two types of data channels are described: logical channel and transport one. The logical channel resides between RLC and MAC layers, while the transport one is located between MAC and physical layers. Furthermore, two types of data channels are also described in the transport channel: dedicated channel and common one. After frames are transmitted from the RLC layer, they first arrive at each MAC-d sublayer via a logical channel, and they are then transmitted to each corresponding UE via one of two types of transport channels. In the case that the dedicated channel is used, they are successively forwarded to each corresponding UE. On the other hand, when the common one is used, RNC schedules their transmission to multiplex them into one shared channel, and then forwards them in turn to each corresponding UE.

Thus, when frames are transmitted via the common channel, the congestion of the MAC layer would more frequently happen since they are multiplexed into one shared channel. When RNC recognizes it by means of feedback information sent from the MAC layer, it controls the transmission rate of the RLC layer to avoid the congestion. The functions of RLC/MAC layers are described in [8][9]. 


\subsection{Proposed Channel Switching Scheme}

In the channel selecting/switching method described in [9], a transmission channel is selected by RNC adaptively based upon the queue length of the RLC dedicated buffer; two thresholds, upper threshold $(T H U)$ and lower threshold $(T H L)$, are employed for that purpose. The detail is described in the following:

- If the queue length of the RLC dedicated buffer exceeds the predetermined upper threshold $T H U$, the transmission channel is switched from the common channel to the dedicated one.

- If the queue length of the RLC dedicated buffer falls below the predetermined lower threshold $T H L$, the transmission channel is switched from the dedicated channel to the common one.

According to the above method, we propose specific channel selecting/switching schemes in the following. The following schemes first assign the common channel for frame transmission to gain the statistical multiplexing effect. However, they are different from each other in how to begin transmitting frames on the dedicated channel after selecting it instead of the common one.

- Scheme 1: Frames currently stored in the RLC dedicated buffer are kept waiting until all frames stored in the MAC-d dedicated buffer are transmitted on the common channel. This assumes that the MAC-d dedicated buffer for each flow is equipped to multiplex frames on the common channel so that those frames cannot be transmitted on the dedicated one.

- Scheme 2: Frames currently stored in the RLC dedicated buffer are immediately transmitted regardless of whether there are any frames stored in the MAC-d dedicated buffer or not. This scheme is also based upon the above assumption. There are possibilities that the related flow uses both the common channel and dedicated one at the same time until all frames in the MAC-d dedicated buffer are transmitted.

- Scheme 3: Frames currently stored in the MAC-d dedicated buffer are immediately transmitted on the dedicated channel assigned now, and then frames stored in the RLC dedicated buffer are successively forwarded to the MAC-d dedicated one. This scheme is free from the above assumption. Hence, the channel switching entity follows the MAC-d dedicated buffer.

\section{Simulation Model}

In this section, we describe our model for simulation to evaluate the channel selecting/switching schemes presented in Sec. 2 We add some modifications to Network Simulator NS Version 2 developed by VINT Project [10] and use it for our research.

\subsection{RLC/UE Model}

Fig. 3] shows the simulation model of RLC layers and UE; Fig. 3. (a) illustrates the model for Scheme 1 and 2, while Fig. 3)(b) describes that for Scheme 3. In RNC, each RLC layer is equipped with a dedicated buffer of $B_{R}$ [frame] and each MAC-d sublayer is equipped with a dedicated buffer of $B_{M}$ [frame]. The buffer size $B_{R}$ and $B_{M}$ is fixed 


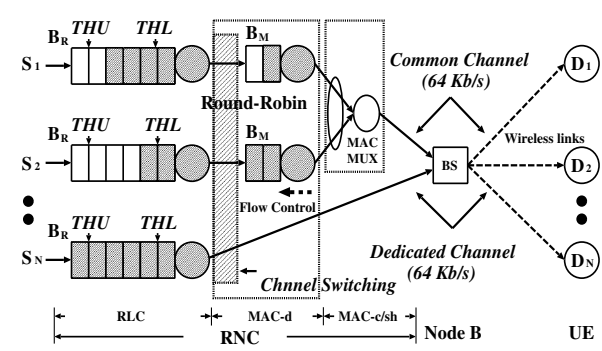

(a) Scheme 1, Scheme 2

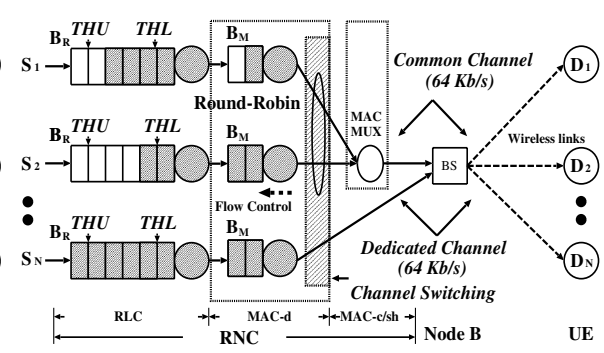

(b) Scheme 3

Fig. 3. Simulation model for channel selecting/switching schemes

at 10 and 2, respectively. One common channel of $64 \mathrm{~Kb} / \mathrm{s}$ is used here, in which the round-robin scheduling algorithm is employed to multiplex frames in several MAC-d dedicated buffers. In this case, to avoid the congestion of the MAC layer, the following flow control scheme based on the queue length of the MAC-d dedicated buffer is adopted:

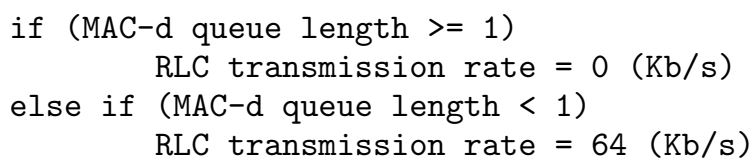

Note that by adopting this scheme, the MAC-d buffer overflow never occurs. Furthermore, several dedicated channels are employed, each of which is of $64 \mathrm{~Kb} / \mathrm{s}$.

\subsection{Traffic Model}

We assume that each traffic source $S_{1}-S_{N}$ transmits web traffic to $D_{1}-D_{N}$, where $N$ is the number of traffic sources. Each source generates traffic according to on/off process. The duration for which frames are successively transmitted is denoted by $T_{O N}$ [frame]. It follows an exponential distribution and the mean length of which is 10 frames of 42 bytes in size. We assume that retransmitted frames are included in $T_{O N}$. The duration for which frames are not transmitted is denoted by $T_{\text {of } f}$ [frame] and it varies according to the amount of traffic denoted by $\lambda$; i.e., $\lambda=N \times T_{O N} /\left(T_{O N}+T_{O F F}\right)$. Note that the total amount of traffic $\lambda=1.0$ indicates a traffic of $64 \mathrm{~Kb} / \mathrm{s}$.

The traffic model is limited so that there may be difficulties in deriving general conclusions. Additionally, we do not consider the wireless aspect of the system in terms of the channel error. However, we here focus on the channel selecting/switching schemes, and our major purpose is to get their fundamental performance.

\section{Numerical Results and Discussions}

In this section, we will evaluate the performance of proposed three schemes for channel selecting/switching by using our simulation model presented in the previous section. For each of these schemes, we first investigate the impact of two thresholds $T H U$ and $T H L$ 


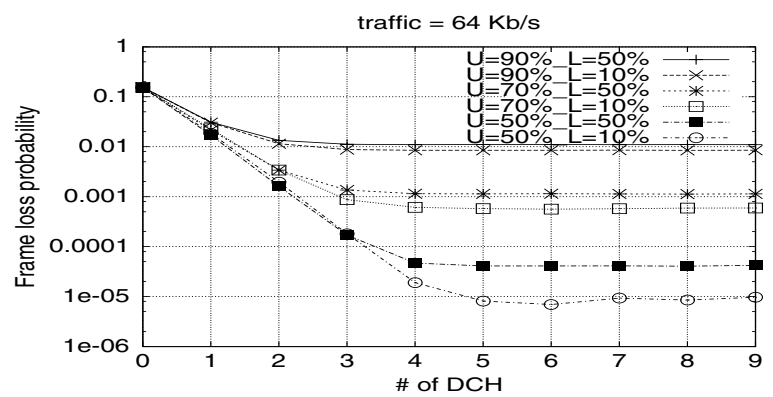

Fig. 4. Frame loss probability (Scheme 1)



Fig. 5. Dedicated channel utilization (Scheme 1)

of the RLC dedicated buffer on the frame loss probability as a function of the maximum number of dedicated channels, which can be utilized by all traffic sources. We then investigate the utilization of the dedicated channel, which is defined by the ratio of the number of frames transmitted via dedicated channels to that of all frames received at each UE. The aim of the proposed schemes is to effectively transmit frames via the common channel and to decrease the utilization of the dedicated channel. Furthermore, by showing the impact of the number of sources and the amount of traffic on the performance, we discuss the optimal number of dedicated channels.

\subsection{Evaluation of Scheme 1}

In this subsection, we focus on the performance of the channel switching Scheme 1 . We set the total amount of traffic arriving at Node $\mathrm{B}, \lambda$, to 1.0 , namely, $64 \mathrm{~Kb} / \mathrm{s}$ and the number of traffic sources, $N$, to 10 .

Fig. 4 shows the frame loss probability of the RLC dedicated buffer. From this figure, we can find that the frame loss probability increases with threshold $T H U$. In this scheme, even if the queue length of the RLC dedicated buffer for a flow exceeds $T H U$ and the dedicated channel is then assigned to the flow, they will be never transmitted until all frames stored in the corresponding MAC-d dedicated buffer are transmitted via the common channel; this is HOL (Head-of-Line) blocking effect. Therefore, if $T H U$ is set to a large value, most newly arriving frames at the RLC dedicated buffer will be lost. 


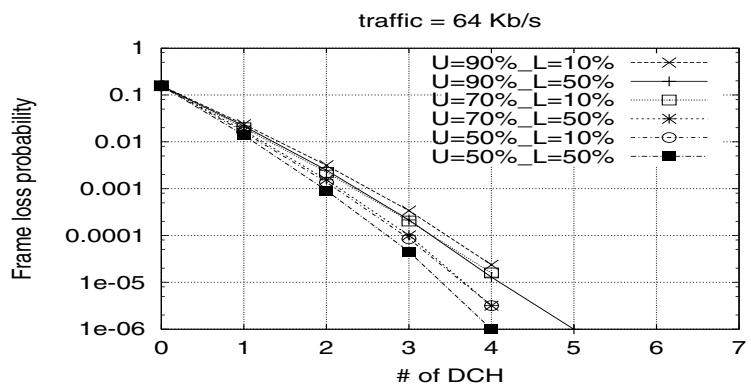

Fig. 6. Frame loss probability (Scheme 2)

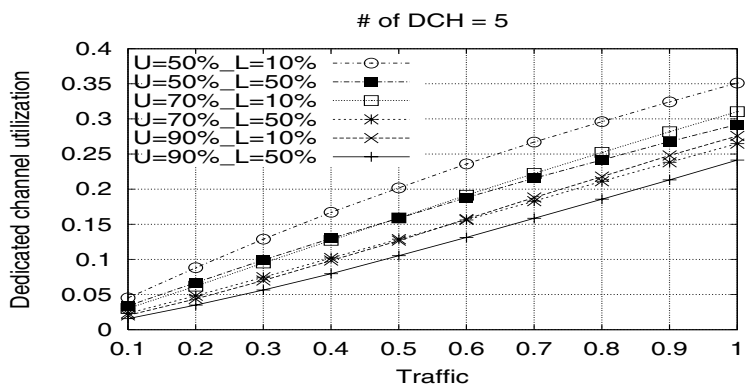

Fig. 7. Dedicated channel utilization (Scheme 2)

If we increase $T H L$ while keeping $T H U$ at a fixed value, frames in the RLC dedicated buffer will be more frequently transmitted via the common channel and HOL blocking will often occur. Thus, the frame loss probability increases with $T H L$. Although the increase of the dedicated channels can basically contribute to the improvement in the frame loss probability, it is limited due to HOL blocking, as shown in Fig. 4

Fig.5 shows the utilization of the dedicated channel as a function of the total amount of traffic at Node B, $\lambda$, when the number of dedicated channels is five. When both $T H U$ and $T H L$ are set to smaller values, the utilization gets larger. The difference in the utilization for different $T H U \mathrm{~s}$ ( $T H L \mathrm{~s}$ ) is almost insensitive to $\lambda$.

Throughout these results, we can say in this scheme that when both THU and THL are set to larger values, frames could be more frequently transmitted on the common channel, whereas the frame loss probability is increasing due to HOL blocking.

\subsection{Evaluation of Scheme 2}

In this subsection, we focus on the performance of the channel switching Scheme 2. We set the total amount of traffic, $\lambda$, to 1.0 and the number of sources, $N$, to 10 .

Fig. [- shows the frame loss probability in this scheme. Unlike in Scheme 1, as shown in Fig. 4 the frame loss probability is not so sensitive to values of the thresholds used and monotonously decreases with the number of dedicated channels. The reason is that Scheme 2 eliminates HOL blocking due to frames in the MAC-d dedicated buffer for 
the common channel by allowing use of both dedicate channel and common one at the same time. For example, in order to achieve the loss probability of less than $10^{-5}$, we should provide at least five dedicated channels.

Fig. 7 shows the utilization of the dedicated channel when five dedicated channels are available. The characteristics shown there are very similar to that of Scheme 1, as shown in Fig. 5] so that Schemes 1 and 2 do not make a large difference to the utilization.

\subsection{Impact of Out-of-Order Transmission}

As mentioned in Sec.2.1 a link layer ARQ protocol is adopted in RNC for transmission error recovery. In this protocol, a receiver will ask the sender to retransmit erroneous frames if necessary. In Scheme 2, frames currently stored in the RLC dedicated buffer are immediately transmitted via the dedicated channel without waiting until all frames stored in the MAC-d dedicated buffer are cleared. Therefore, at the receiver frames transmitted via the common channel may be overtaken by ones transmitted via the dedicated channel, which is referred to as out-of-order transmission since the common channel is shared by several flows, e.g., in a round-robin basis. This will further cause unnecessary retransmission request, resulting in a wasteful use of radio resources. Thus, we will discuss the impact of out-of-order transmission on retransmission property when five dedicated channels are employed. We will define an out-of-order as a case when the frames transmitted over the common channel are overtaken ones over the dedicated channel, and call such overtaken frames out-of-order ones. Furthermore, we define the number of successively overtaken frames as the number of frames transmitted over the dedicated channel until a frame in the MAC-d dedicated buffer is transmitted when out-of-order transmission happened.

Out-of-order Probability. We investigate the out-of-order probability as shown in Fig. 8 in case where $N=10$ and the number of dedicated channels equals five. We define it as a ratio of the number of out-of-order frames to that of the total received frames at UE. We can see from this figure that the probability increases with $T H L$ because the transmission channel more frequently changes from the dedicated one to the common one and vice versa. Fig. 9 shows the probability of the number of successively overtaken frames during an out-of-order transmission. When both $T H U$ and $T H L$ get smaller, the probability that the number of overtaken frames is two or more becomes smaller. This would result in less retransmission of overtaken frames.

Retransmission Probability for Out-of-order Transmission. We investigate retransmission probability caused by out-of-order transmission. We define the probability, when the acceptable number of out-of-order frames is $i$, as $p_{o} \times \sum_{k=i+1}^{\infty} p_{o v}(k)$, where $p_{o}$ is the out-of-order probability in Fig. 8 and $p_{o v}(k)$ is the probability given by Fig. 9 when the number of successively overtaken frames is $k$. Fig. 10 shows the retransmission probability. From this result, if receivers can allow five successively overtaken frames, the retransmission probability for out-of-order transmission is less than $10^{-6}$. Thus, we can say in this scheme that setting both $T H U$ and $T H L$ to relatively small value is effective in achieving high throughput.

\subsection{Evaluation of Scheme 3}

In this subsection, we investigate Scheme 3. We set the total amount of traffic, $\lambda$, to 1.0 and the number of sources, $N$, to 10 . 


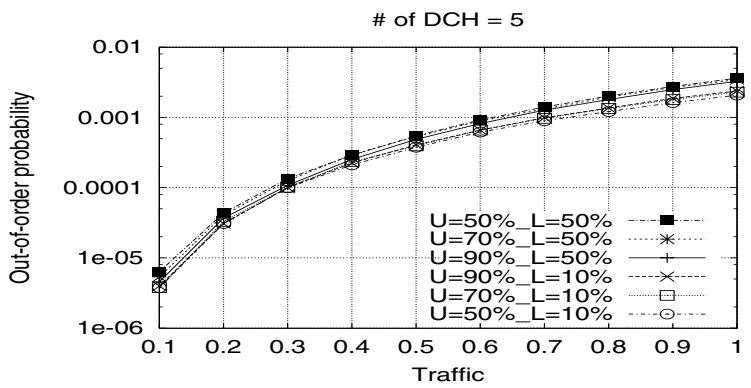

Fig. 8. Out-of-order probability

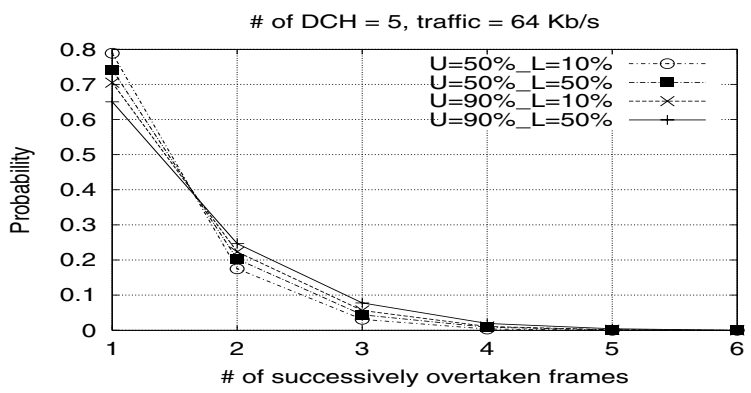

Fig. 9. Probability of the number of successively overtaken frames



Fig. 10. Probability that frames are retransmitted due to out-of-order arrivals

Fig. 11 shows the frame loss probability. From this result, it is almost the same as that of Scheme 2 as shown in Fig. 6. However, unlike in Scheme 2, the our-of-order transmission never occurs at receivers since frames stored in the RLC dedicated buffer are just transmitted after all frames stored in the corresponding MAC-d dedicated buffer are transmitted via the dedicated channel. 


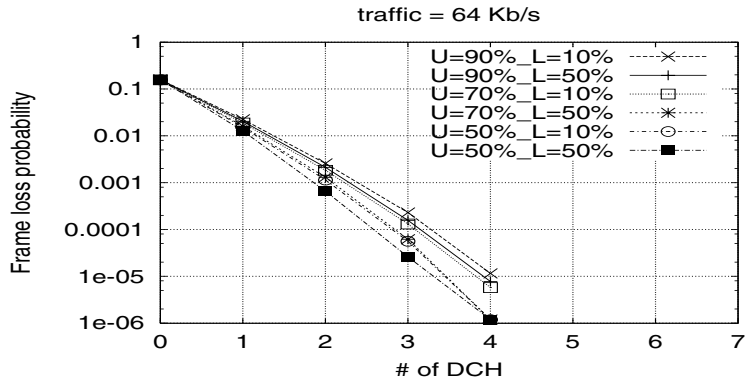

Fig. 11. Frame loss probability (Scheme 3)

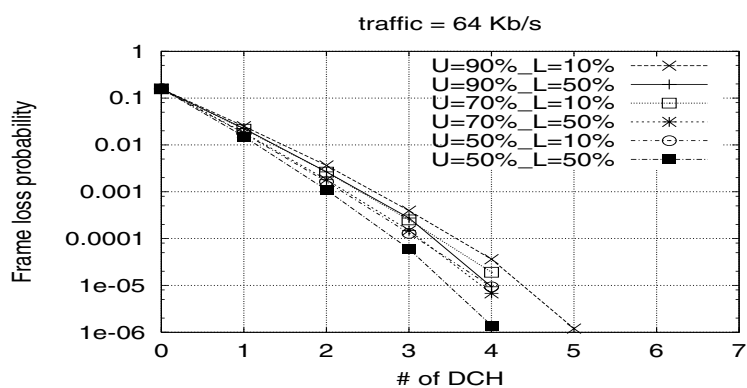

(a) Frame loss probability $(N=20, \lambda=1.0)$

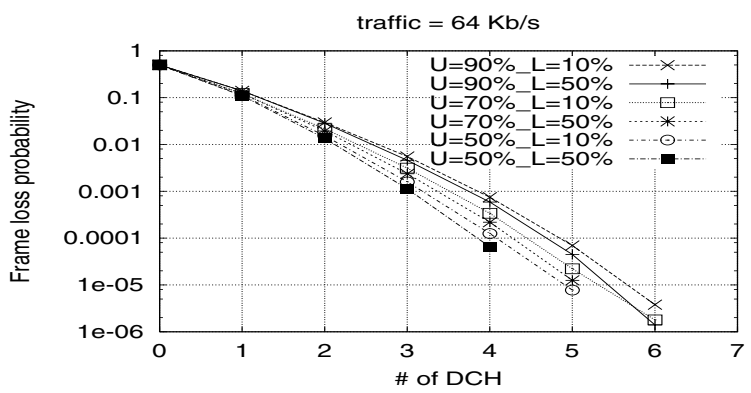

(b) Frame loss probability $(N=10, \lambda=2.0)$

Fig. 12. Impact of the number of sources and the amount of total traffic (Scheme 3)

\subsection{Impact of the Number of Sources and the Amount of Total Traffic}

In this subsection, we investigate the impact of the number of sources and the amount of the total traffic in Scheme 3. 
Table 1. Switching times per second (Scheme 2)

\begin{tabular}{|c|c|c|c|c|}
\hline \multicolumn{5}{|c|}{$L=10 \%$, Scheme 2 } \\
\hline traffic & $N$ & $U=50 \%$ & $U=70 \%$ & $U=90 \%$ \\
\hline $64 \mathrm{~Kb} / \mathrm{s}$ & 10 & 4.3718 & 3.3735 & 2.6458 \\
\hline $64 \mathrm{~Kb} / \mathrm{s}$ & 20 & 4.5017 & 3.4866 & 2.7509 \\
\hline $128 \mathrm{~Kb} / \mathrm{s}$ & 10 & 12.9046 & 10.7725 & 9.3105 \\
\hline \hline \multicolumn{5}{|c|}{$L=50 \%$, Scheme 2} \\
\hline traffic & $N$ & $U=50 \%$ & $U=70 \%$ & $U=90 \%$ \\
\hline $64 \mathrm{~Kb} / \mathrm{s}$ & 10 & 5.0870 & 3.8251 & 2.9754 \\
\hline $64 \mathrm{~Kb} / \mathrm{s}$ & 20 & 5.1810 & 3.9024 & 3.0665 \\
\hline $128 \mathrm{~Kb} / \mathrm{s}$ & 10 & 17.0085 & 13.8733 & 11.6719 \\
\hline
\end{tabular}

Table 2. Switching times per second (Scheme 3)

\begin{tabular}{|c|c|c|c|c|}
\hline \multicolumn{5}{|c|}{$L=10 \%$, Scheme 3 } \\
\hline traffic & $N$ & $U=50 \%$ & $U=70 \%$ & $U=90 \%$ \\
\hline $64 \mathrm{~Kb} / \mathrm{s}$ & 10 & 4.2914 & 3.2893 & 2.6013 \\
\hline $64 \mathrm{~Kb} / \mathrm{s}$ & 20 & 4.4010 & 3.4078 & 2.7106 \\
\hline $128 \mathrm{~Kb} / \mathrm{s}$ & 10 & 12.5823 & 10.5501 & 9.0728 \\
\hline \hline \multicolumn{5}{|c|}{$L=50 \%$, Scheme 3 } \\
\hline traffic & $N$ & $U=50 \%$ & $U=70 \%$ & $U=90 \%$ \\
\hline $64 \mathrm{~Kb} / \mathrm{s}$ & 10 & 4.984 & 3.758 & 2.9343 \\
\hline $64 \mathrm{~Kb} / \mathrm{s}$ & 20 & 5.0627 & 3.8339 & 2.9933 \\
\hline $128 \mathrm{~Kb} / \mathrm{s}$ & 10 & 16.3054 & 13.2505 & 11.2249 \\
\hline
\end{tabular}

Fig. 12 shows the frame loss probability of Scheme 3 when $N=20, \lambda=1.0$ (Fig.12]a)) and $N=10, \lambda=2.0$ (Fig.112(b)). We can see from Fig.112(a) that although $N$ increases to 20, the number of required dedicated channels becomes five and it is the same as that in case when $\lambda=1.0$ and $N=10$ (See Fig. 111). In addition, if $\lambda=2.0$ and $N=10$, the number becomes only six. To further show the effectiveness of Scheme 3 , we will indicate the average number of switching times from the common channel to dedicated one per second in Tables 1 (Scheme 2) and 2 (Scheme 3), when the number of dedicated channels is five $(\lambda=1.0)$ or six $(\lambda=2.0)$. These results are related to the channel switching overhead required. It is found that the results in Scheme 3 are smaller than those in Scheme 2 for some THUs and THLs.

\section{Concluding Remarks}

In this paper, we proposed details of three channel switching schemes according to the specification of RNC in the third-generation mobile telecommunication systems. They are adaptively selecting the appropriate transmission channel from the common channel or the dedicated one in accordance with the queue length of the RLC dedicated buffers. In order to investigate the impact of thresholds in its buffer, we evaluate this performance by simulation. Through numerical results, we have obtained the followings. We first propose Scheme 1 in which the transmission channel switched from the common one to the dedicated one after all frames stored in the corresponding MAC-d dedicated buffer are transmitted via the common channel. Therefore, the frame loss probability of the RLC dedicated buffer cannot be improved by increasing the number of dedicated channels due to HOL blocking effect in MAC-d dedicated buffer.

In Scheme 2, the transmission channel is immediately switched regardless of whether there are any frames stored in the corresponding MAC-d dedicated buffer or not. Thus, the frame loss probability is decreasing with the number of dedicated channels and Scheme 2 is thus more effective than Scheme 1. However, Scheme 2 leads to the out-oforder transmission; i.e., frames via the common channel may be overtaken by ones via the dedicated one, resulting in unnecessary retransmissions. Therefore, a wasteful use of radio resources may occur.

To overcome weak points in Scheme 1 and 2, we also proposed Scheme 3 in which if the transmission of some flows is assigned to the dedicated channels, the corresponding frames in MAC-d dedicated buffer are immediately transmitted via the dedicated channel 
prior to those in the RLC dedicated buffer. This provides good performance in terms of the frame loss probability as in Scheme 2 without causing the out-of-order transmission as in Scheme 2.

We evaluated the performance of RNC in this paper by focusing only on the case of specific traffic model. Thus, we should also investigate the case where various types of traffic at RNCs for the further work.

Acknowledgments. The authors would like to thank Matsushita Communication Industrial Co., Ltd. for many helpful comments, advice, and suggestions. This work was supported in part by Research for the Future Program of Japan Society for the Promotion of Science under the Project "Integrated Network Architecture for Advanced Multimedia Application Systems” JSPS-RFTF97R16301), Telecommunication Advancement Organization of Japan under the Project "Global Experimental Networks for Information Society Project," and a Grant-in Aid for Scientific Research (12450156) of the Ministry of Education, Culture, Sports, Science and Technology, Japan.

\section{References}

1. IMT-2000, http://www.itu.int/imt/.

2. R. D. Chrsello, et al, "IMT-2000 Standards: Radio Aspects," IEEE Personal Communications, vol. 4, no. 4, pp. 8-40, August 1997.

3. M. Zeng, A. Annamalai, and Vijay K. Bhargava, "Harmonization of global third-generation mobile systems," IEEE Communications Magazine, vol. 38, no. 12, pp. 94-104, December 2000.

4. F. Adachi, M. Sawahashi, and H. Suda, "Wideband-CDMA for next-generation mobile communications systems," IEEE Communications Magazine, vol. 36, no. 9, pp. 70-80, September 1998.

5. E. Dahlman, B. Gudmundson, Mats Nilsson, and J. Sköld, "UMTS/IMT-2000 based on Wideband CDMA," IEEE Communications Magazine, vol. 36, no. 9, pp. 56-69, September 1998.

6. 3rd Generation Partnership Project, http://www.3gpp.org/.

7. S. Onoe, K. Ohno, K. Yamagata, and T. Nakamura, "Wideband-CDMA radio control techniques for third-generation mobile communication systems," Proceedings of IEEE Vehicular Technology Conference, pp. 835-839, May 1997.

8. 3GPP TS 25.322 V4.0.0 RLC protocol specification, March 2001.

9. 3GPP TS 25.321 V4.0.0 MAC protocol specification, March 2001.

10. VINT Project Network Simulator ns-2, http://www.isi.edu/nsnam/ns/. 